\title{
A pursuit problem in an infinite system of second-order differential equations
}

\begin{abstract}
We study a pursuit differential game problem for an infinite system of second-order differential equations. The control functions of players, i.e., a pursuer and an evader are subject to integral constraints. The pursuit is completed if $z(\tau)=z^{\cdot}(\tau)=0$ at some $\tau>0$, where $\mathrm{z}(\mathrm{t})$ is the state of the system. The pursuer tries to complete the pursuit and the evader tries to avoid this. A sufficient condition is obtained for completing the pursuit in the differential game when the control recourse of the pursuer is greater than the control recourse of the evader. To construct the strategy of the pursuer, we assume that the instantaneous control used by the evader is known to the pursuer.
\end{abstract}

Keyword: Pursuit problem; Infinite system of second-order differential equations 